\title{
Computer studies of the dynamic strength of ceramics (II)
}

\author{
D.J. Steinberg \\ Lawrence Livermore National Laboratory, P.O. Box 808 (L35), Livermore, CA 9455I, U.S.A.
}

\begin{abstract}
Résumé: Nous avonsétendu notre loi de comportement pour céramiques complètement compactes. Maintenant que ce modèle comprend $\mathrm{Al}_{2} \mathrm{O}_{3}$ (AD999) et $\mathrm{Si}_{3} \mathrm{~N}_{4}$, nous possèdons des paramètres pour sept matériaux. Nous avons simulé avec succès des résultats nouveaux d'essais, tels que l'écaillage et les expériences de double-choc pour $\mathrm{SiC}$ et $\mathrm{B}_{4} \mathrm{C}$. Nous proposons aussi des expériences supplémentaires qui doivent elucider les problèmes restants.
\end{abstract}

\begin{abstract}
Our previous constitutive model for fully dense ceramics has been extended to include $\mathrm{Al}_{2} \mathrm{O}_{3}$ (AD999) and $\mathrm{Si}_{3} \mathrm{~N}_{4}$; there are now parameters for seven materials. New experimental data, such as spall and double-shock wave-profiles for $\mathrm{SiC}$ and $\mathrm{B}_{4} \mathrm{C}$, have been successfully simulated. Additional experiments are proposed which should help elucidate the remaining problems.
\end{abstract}

\section{INTRODUCTION}

In a previous article, (hereafter referred to as [1]), I described a new constitutive model for the dynamic strength of fully-dense ceramics. The purpose of this present report is to add information regarding new materials and to make additions and corrections to the old data base. In particular, I discuss double-shock experiments, spall, data for yield strength vs. strain rate, and ultrasonic data for the pressure dependence of the shear modulus. For a complete description of the constitutive model, the Mie-Grüneisen equation of state, as well as the definitions of all terms and symbols, the reader is referred to [1].

\section{EQUATION OF STATE AND DYNAMIC STRENGTH}

$2.1 \mathrm{Al}_{2} \mathrm{O}_{3} \quad$ Grady has performed two successful VISAR experiments on AD999 at 53- and 63-GPa peak stress [2]. AD999 is an alumina with only $0.6 \%$ porosity and a Hugoniot elastic limit (HEL) of $12 \mathrm{GPa}$. These data are compared in Fig. 1 with hydrocode simulations using the parameters in Table 1. As in [1], all data for initial density $\rho_{0}$, bulk sound speed $C_{0}$, and shear modulus $G_{0}$ are taken from Grady's work. The Grüneisen gamma $\gamma_{0}$ and rate-independent yield strength $Y_{A}$ were determined by the author. To achieve the calculated results shown, the entire experiment, including the 6061-T6 Al sabot, had to be included. The equations of state (EOS) and constitutive models for $\mathrm{Al}, \mathrm{PMMA}, \mathrm{Ta}$, and $\mathrm{W}$, the latter two rate-independent, are found in [3]. Except for an unexplained vertical offset, the agreement in the timing and shape between the experimental and calculated profiles is excellent. Even many small details in the shape of the experimental profiles are faithfully reproduced in the calculations.

The experimental time difference between the elastic and plastic waves, as well as the peak velocity, are also very well reproduced in the calculations. In previous work with AD995, the experimental time difference was always significantly longer and the peak lower than in the calculations, consistent with the fact that AD 995 is about $2.4 \%$ porous. In addition, the shape of the calculated and experimental loading profiles are in good agreement which adds to the credence of the rate-dependent part of the model. Finally, the experimental and calculated initial release times agree, in accord with the ultrasonically-measured pressure dependence of $G$. 


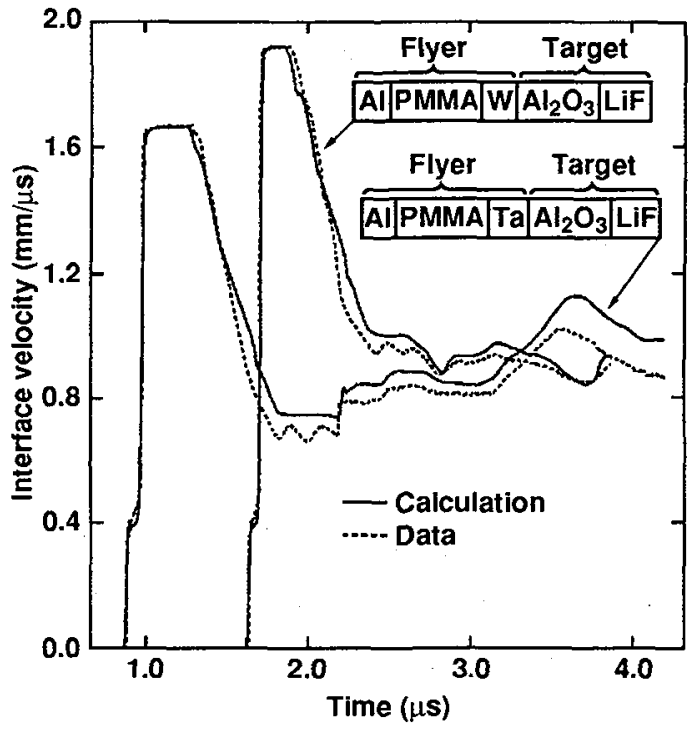

Fig. 1. Comparison of experiment and calculation for $\mathrm{Al}_{2} \mathrm{O}_{3}$ shocked to 53 and $63 \mathrm{GPa}$.

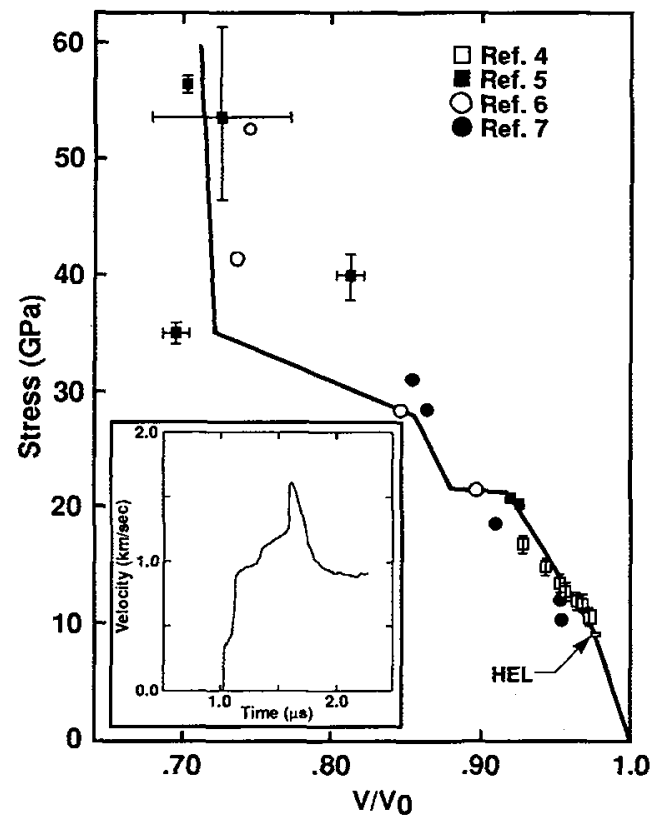

Fig. 2. Qualitative sketch of the Hugoniot compared with the available data for AIN. The insert is a 1D wave profile showing two breaks.

However, a major discrepancy occurs at the end of the first or principal release where several oscillations in the data are not reproduced in the calculations. At first glance these oscillations appear to be spall signals. However, at this time the calculation shows that the alumina is not in tension. This is true even when the density of the PMMA was arbitrarily cut in half to force the calculations to fall below the data. This would imply that spall is not the origin of these oscillations. In addition, if spall were present, it seems very unlikely that it would not have affected the later part of the signal, especially the timing. I have no explanation for these oscillatory structures. However, it is important to emphasize that their presence in experimental data does not necessarily mean that spall has occurred.

2.2 AlN Figure 2 shows a qualitative sketch of the Hugoniot compared with the available data for AlN [47]. With the exception of the point at a relative volume of 0.81 , the picture is consistent with two separate phase transformations, one at $21.5 \pm 1 \mathrm{GPa}$ and a relative volume of about 0.92 , and the other at $28 \pm 1.5 \mathrm{GPa}$ and a relative volume of approximately 0.85 . The first transformation is accompanied by a volume change of about $4 \%$; the second, about $13 \%$. Of course, the second break may also be due, in full or in part, merely to wave

Table 1. Material parameters.

\begin{tabular}{|c|c|c|c|c|c|c|c|c|c|c|c|c|c|}
\hline Material & $\frac{\rho_{0}}{\mathrm{~g} / \mathrm{cm}^{3}}$ & $\frac{C_{0}}{\mathrm{~mm} \mu \mathrm{s}}$ & $s_{1}$ & $\gamma_{0}$ & $\frac{G_{0}}{\mathrm{GPa}}$ & $\frac{Y_{\mathrm{A}}}{\mathrm{GPa}}$ & \multicolumn{2}{|c|}{$\mathrm{TPa}^{-1}$} & & $10^{-4} \mathrm{~K}^{-1}$ & $\frac{{ }^{c} D}{\mathrm{MPa}^{\mathrm{n}}}$ & $\frac{{ }^{\mathrm{d}} D_{\text {est }}}{\mathrm{MPas}^{1 / 3}}$ & $\frac{K_{\mathrm{IC}}}{\mathrm{MPam}^{1 / 2}}$ \\
\hline $\mathrm{SiC}$ & 3.177 & 8.19 & 0.88 & 1.16 & 186.9 & 12.0 & 12. & 12. & - & 1.0 & 22. & 12. & 4.4 \\
\hline PSZ & 6.028 & 5.67 & 1.0 & 1.57 & 83.4 & 6.0 & 30. & 16. & - & 3.4 & 9.5 & 19. & 8.25 \\
\hline $\mathrm{B}_{4} \mathrm{C}$ & & & & & & & & & & & & & \\
\hline$E-P$ & 2.516 & 9.57 & 1.0 & 1.46 & 199.3 & 11.1 & 11. & 14. & - & 3.0 & 14. & 7.5 & 2.4 \\
\hline Dow & 2.506 & 9.65 & 1.0 & 1.46 & 197.2 & 12.1 & 11. & 14. & - & 3.0 & 14. & 7.5 & 2.4 \\
\hline $\mathrm{TiB}_{2}$ & 4.452 & 6.96 & $-{ }^{e}$ & 1.39 & 237.2 & 10.1 & 12. & 14.5 & 0.94 & 1.0 & 14. & 13. & 4.6 \\
\hline AlN & 3.257 & 7.87 & $0-0.4$ & 0.65 & 130.3 & 6.0 & - & 6.5 & - & 1.5 & 9.5 & 12. & 4.5 \\
\hline $\mathrm{Al}_{2} \mathrm{O}_{3} \mathrm{f}$ & 3.948 & 7.97 & $1.27 \mathrm{~g}$ & 1.31 & 160.7 & 7.1 & $11 .^{\mathrm{h}}$ & 11. & $1,2^{\mathrm{i}}$ & 1.2 & 9.5 & 12. & $4.0^{\mathrm{j}}$ \\
\hline $\mathrm{Si}_{3} \mathrm{~N}_{4}$ & 3.156 & 7.83 & 1.0 & 0.61 & 106.5 & 4.6 & 6. & 6. & $1.1^{\mathrm{k}}$ & 1.1 & 22. & 14. & $5.9^{1}$ \\
\hline
\end{tabular}


interactions. This Hugoniot sketch is also in qualitative agreement with several experimental wave profiles from Grady [2], which show pronounced, step-like changes in the shape of the loading profile at velocities appropriate to these stress levels (e.g., the insert in Fig. 2). Grady's profiles also show evidence of a strong shock up to the first transformation. It is difficult to reconcile this with the concave downward shape in the data of Rosenberg et al. [4]. However, with the exception of the highest stress point, the experimental error in these data could accommodate a concave upward shape.

Because the model lacks a phase-change mechanism, I have not shown any comparisons of calculations with Grady's data for peak stresses above $21.5 \mathrm{GPa}$. Unfortunately, the only reliable low-stress profiles are at or below the HEL, and these really do not provide a stringent test for the model. There is also some question about the quality of the AlN tested [8]. I suggest that a few new profiles below $21.5 \mathrm{GPa}$, but well above the HEL, would be good tests for the model.

$2.3 \mathrm{TiB}_{2}$ Following a suggestion made in [1], diamond-anvil pressure-cell experiments were performed by J. Akella in an effort to elucidate the origin of the so-called double yield [9]. This feature has been observed in wave profiles taken on material from four different suppliers [8]. The x-ray diffraction measurements were made in a hydrostatic environment to $12 \mathrm{GPa}$, and quasi-hydrostatic to $40 \mathrm{GPa}$ with no evidence of any phase change until approximately $30 \mathrm{GPa}$. As a follow-on to this work, I suggest that at least one plate-impact experiment be performed with a ramp-wave generator. This will allow us to see if the existence of the double yield depends on a high rate of stress loading.

$2.4 \mathrm{~B}_{4} \mathrm{C}$ Grady has produced two new wave profiles on $\mathrm{B}_{4} \mathrm{C}$ at peak stresses of 42.2 and $60.5 \mathrm{GPa}$ [2]. The lower stress experiment had a Ta impactor, and, as usual, employed a VISAR to measure the velocity of the interface between the target and a LiF window. The higher stress experiment was a symmetrical impact. Both experiments were at higher stresses than his previous work with Dow material [2]. The new profiles, especially the higher stress one, show much less evidence of the strong oscillations that characterized all the earlier, lower stress data [1]. In fact these profiles are at a high enough peak stress that the limit on rate dependence described in [1] is operative. The hydrocode simulations were done with this limit $Y_{L}$ set to $1.5 \mathrm{GPa}$.

Figure 3 compares the 42.2-GPa profile with a hydrocode simulation using the model described in [1]. The agreement is satisfactory with the very important exception of the time difference between the elastic and plastic loading waves. Substantially increasing parameter C from [1] did not alleviate the problem. There is a similar, but smaller, discrepancy for the 60.5-GPa comparison.

For the previous lower stress data, it was often impossible to see the arrival of the main shock. However, the first reflection of the elastic precursor off the oncoming main shock was always visible, and this gives a good relative measure of the shock velocity. Figure 4 shows the early-time history for all the experiments done with the Dow material. In order to compare these results, they have all been normalized to the same target thickness. It is clear that up to $30 \mathrm{GPa}$, the first reflection arrival times imply a continuous increase in shock velocity as the peak stress increases. However, at $42.2 \mathrm{GPa}$, this arrival time is later than for the 25.7-GPa

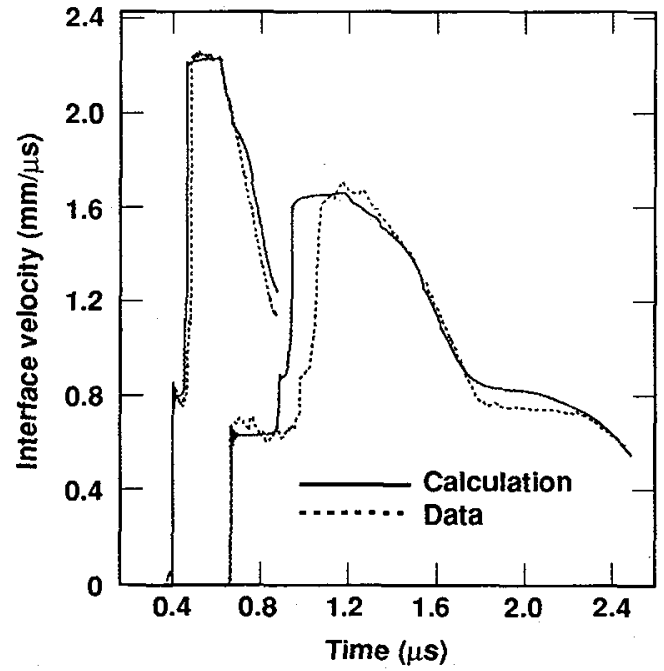

Fig. 3. Comparison of experiment and calculation for $\mathrm{B}_{4} \mathrm{C}$ shocked to 42.2 and $60.5 \mathrm{GPa}$.

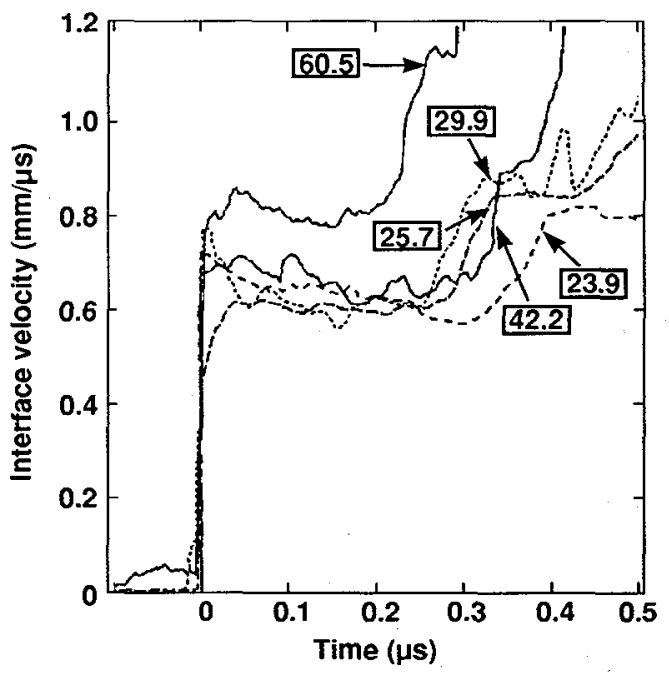

Fig. 4. Comparison of the experimental arrival times for the first elastic reflection for the Dow $B_{4} C$. 
experiment, implying that the shock velocity is anomalously slow compared with the lower stress data. It is not at all clear what these results mean. The original model fit all the Hugoniot data up to $100 \mathrm{GPa}$ ([1], Fig. 10). While there is a hint of a possible phase change in these data, no evidence of one is seen in the wave profiles.

Another unexplained problem can also be seen in Fig. 4. The HEL for the 60.5-GPa experiment is approximately $30 \%$ higher than the HEL for the other profiles. (To get the agreement between the simulation and the experiment in Fig. 3, $Y_{A}$ was increased from 12.1 to $15.1 \mathrm{GPa}$.) Grady has suggested that this difference could be related to precursor decay as the target thickness in this experiment was about $1 / 3$ that of the others [8]. I feel, however, that the difference is too great for this explanation to be valid.

I would suggest that the region between 30 and $42 \mathrm{GPa}$ be explored with additional wave profiles, all taken using one well-characterized batch of material.

$2.5 \mathrm{Si}_{3} \mathrm{~N}_{4}$ As has been the case with all other ceramics, $\mathrm{Si}_{3} \mathrm{~N}_{4}$ has its own individual peculiarities. This can be seen in the wave profiles from Grady [2]. In Fig. 5, Grady's lowest stress profile is compared with a hydrocode simulation using the parameters in Table 1. It is quite clear that the principal experimental shockwave velocity is much slower than the calculation. Indeed, this velocity does not exceed the bulk sound speed even when the peak driving stress is about $43 \mathrm{GPa}$. However, the insert in Fig. 5 shows that a small peak occurs immediately after the HEL which is apparent in three out of four profiles. This early structure occurs at a time that is approximately coincident with a calculation which uses a shock velocity-particle velocity slope of 1.0; however, this value is much different from the value of 1.55 given by Mashimo et al. [10]. This peak is largest and most obvious in the lowest stress experiment, being about $12 \mathrm{GPa}$. This would suggest that additional experiments should be done at peak driving stresses between the HEL ( $9.4 \mathrm{GPa}$ ) and about $15 \mathrm{GPa}$ to define this feature further. Perhaps a phase transition has taken place at about $12 \mathrm{GPa}$. Also, experimental sources for this effect have not yet been ruled out [8].

\section{DOUBLE-SHOCK EXPERIMENTS}

Grady and co-workers have performed double-shock experiments on $\mathrm{SiC}$ [11] and $\mathrm{B}_{4} \mathrm{C}$ [2]. Both employed a composite flyer consisting of $\mathrm{LiF}$ backed by Ta. These data are compared with hydrocode simulations in Fig. 6. For $\mathrm{SiC}$, we used the same model as described in [1]. The agreement could hardly be improved upon. In particular, the calculated arrival time and maximum velocity of the elastic precursor at reshock are in excellent agreement with the data. Because I estimate that $2 \mathrm{D}$ effects should start around $2.1 \mu \mathrm{s}$ after the arrival of the elastic wave, the agreement beyond $3.4 \mu$ s may be fortuitous.

For $\mathrm{B}_{4} \mathrm{C}$, the model described in [1] was used with the exception that the value of $\mathrm{Y}_{\mathrm{A}}$ was reduced from 12.1 to $11.6 \mathrm{GPa}$. This change brought the calculated velocities at the HEL and first elastic reflection into

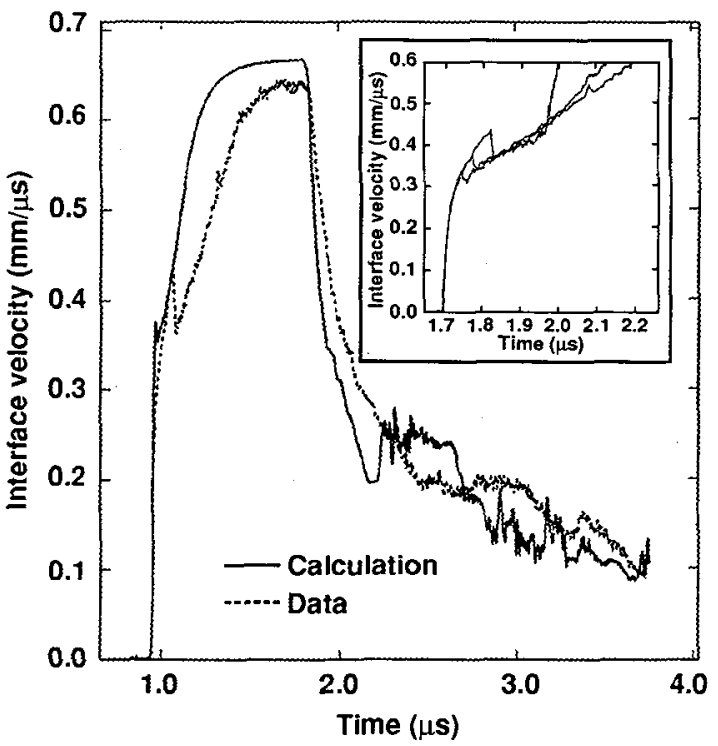

Fig. 5. Comparison of experiment and calculation for $\mathrm{Si}_{3} \mathrm{~N}_{4}$ shocked to about $15 \mathrm{GPa}$. The insert shows the early-time history for 3 profiles with different final loading stress.

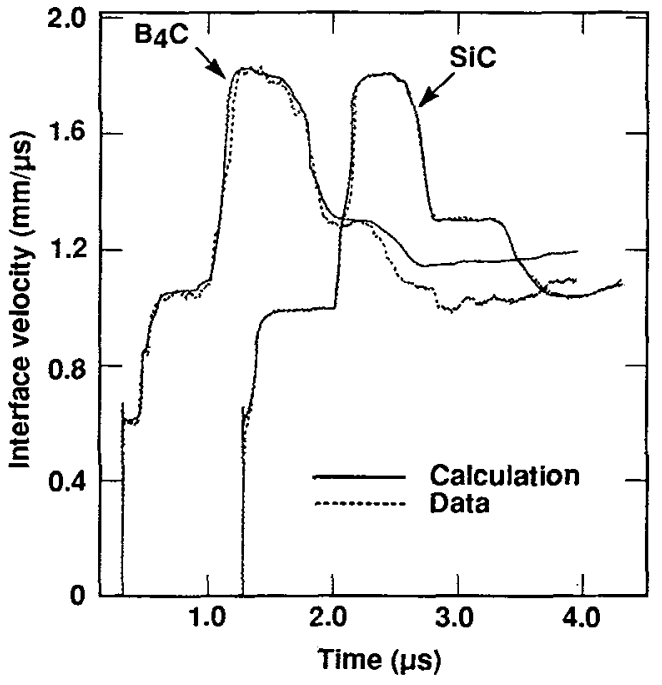

Fig. 6. Comparison of experiment and calculation for $\mathrm{SiC}$ and $\mathrm{B}_{4} \mathrm{C}$ which have been doubleshocked. 
agreement with the data, but did affect the later parts of the simulation. Grady has noted that there is enough sample-to-sample variation in $\mathrm{B}_{4} \mathrm{C}$ to easily account for this few percent spread in HEL [8]. I estimate that 2D effects should commence at about $2.2 \mu \mathrm{s}$, which is where the calculation and data begin to differ. Besides calculating the correct arrival time of the elastic precursor at reshock, one can see that the calculation simulates some rather fine details very well. Note particularly the small structure just before the arrival of the elastic precursor at reshock as well as some fine structures at the reshock peak and release.

\section{SPALL}

Grady and his co-workers have performed plate-impact experiments to determine the spall strength of a number of ceramics $[2,11,12]$. The analysis of these data, given in Table 2 , includes calculated maximum stress $\sigma_{\mathrm{m}}$, the ratio (in $\%$ ) of $\sigma_{\mathrm{m}}$ to the HEL, and an estimate of strain rate $\dot{\varepsilon}$ during the spall process. The spall strength $\sum$ was determined using the Cochran-Banner model [13] in a 1D Lagrangian hydrocode simulation of the experimental wave-profiles. The strain rate was determined from the experimental profiles by the fractional change in velocity $\left(v_{\max }-v_{\min }\right) / v_{\max }$ divided by the time difference from $v_{\max }$ to $v_{\min }$. Here, $v_{\max }$ $-v_{\min }$ is identical to the well-known pullback velocity.

For most materials the spall strength is about $0.5 \mathrm{GPa}$ when the strain rate is approximately $3 \mu \mathrm{s}^{-1}$. In one case $\left(\mathrm{B}_{4} \mathrm{C}\right)$, where the rate is about 10 times greater, $\Sigma$ is $1.4 \mathrm{GPa}$. Spall strength usually increases with strain rate, so qualitatively, at least, this increase is reasonable. For AlN, there is one experiment where $\sigma_{\mathrm{m}}$ slightly exceeds the HEL, and in this case, $\sum$ is smaller than it is in an experiment performed below the HEL. This is in agreement with conventional wisdom, but even the existence of a spall strength above the HEL is still an open question.

The most intriguing material is PSZ which (Fig. 7) exhibits a spall strength of 2 GPa [12]. This is a higher value than that of most metals, certainly brittle ones like tungsten [3]. It would be highly informative to perform a few experiments on this material where $\sigma_{\mathrm{m}}$ exceeds the HEL by 20 to $50 \%$.

\section{PRESSURE DEPENDENCE OF THE SHEAR MODULUS}

Table 1 compares the results for $[\mathrm{d}(\ln G) / \mathrm{d} P]_{0}$ for an estimated value, the formula for which is described in [14], and a value determined from the elastic release time in the measured wave profiles. The latter can be considered as a valid experimental approach. The assumptions required are as follows: (1) The temperature dependence of $G$ is either known or, if not, has only a small effect on $G$ as compared with pressure; (2) a MieGrüneisen equation of state, referenced to the Hugoniot, provides an adequate representation of the bulk modulus and its variation with pressure; (3) an ensuing hydrocode simulation of the wave profile basically reproduces the experimental data. All of these assumptions I believe are valid.

For $\mathrm{Al}_{2} \mathrm{O}_{3}$, the two values also agree with one measured by ultrasonic techniques [15]. Presently, groups led by Prof. M. Manghnani at the University of Hawaii and by Dr. D. Dandekar at the Army Research Laboratory, Watertown, MA, are working on additional ultrasonic measurements.

Table 2. Spall strengths.

\begin{tabular}{lcccc}
\hline Material & $\begin{array}{c}\sigma_{\mathrm{m}} \\
(\mathrm{GPa})\end{array}$ & $\begin{array}{c}\sigma_{\mathrm{m} / \mathrm{HEL}} \\
(\%)\end{array}$ & $\begin{array}{c}\Sigma \\
(\mathrm{GPa})\end{array}$ & $\begin{array}{c}\dot{\varepsilon} \\
\left(\mu \mathrm{s}^{-1}\right)\end{array}$ \\
\hline $\mathrm{SiC}$ & 12.0 & 69 & 0.5 & 15 \\
$\mathrm{PSZ}$ & 12.7 & 78 & 2.0 & 4 \\
$\mathrm{PSZ}$ & 11.6 & 72 & 2.0 & 4 \\
$\mathrm{TiB} 2$ & 9.3 & 67 & 0.5 & 2 \\
$\mathrm{TiB} 2$ & 6.9 & 50 & 0.5 & 4 \\
$\mathrm{TiB} 2$ & 2.9 & 21 & 0.5 & 5 \\
$\mathrm{~B}_{4} \mathrm{C}$ & 6.6 & 39 & 1.4 & 38 \\
$\mathrm{~B}_{4} \mathrm{C}$ & 4.9 & 29 & 0.5 & 6 \\
$\mathrm{~B}_{4} \mathrm{C}$ & 3.6 & 21 & 0.5 & 5 \\
$\mathrm{AlN}$ & 4.9 & 52 & 0.7 & 3 \\
$\mathrm{AIN}$ & 10.1 & 108 & 0.4 & 7 \\
\hline
\end{tabular}

$\mathrm{B}_{4} \mathrm{C}$ is Dow material; high $\mathrm{HEL}$ for $\mathrm{TiB}_{2}$

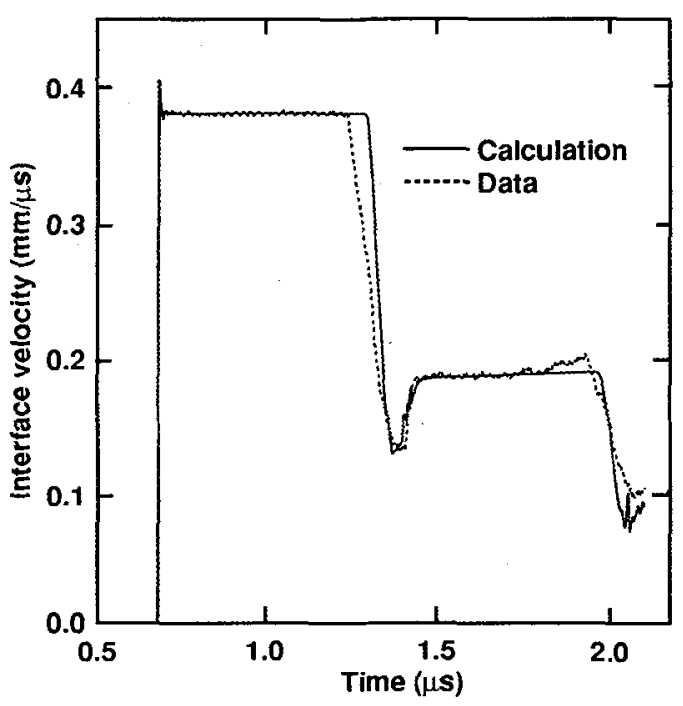

Fig.7. Comparison of experiment and calculation showing spall in PSZ. 


\section{YIELD STRENGTH VS. STRAIN RATE}

Following a suggestion made in [1], J. Lankford measured the variation in yield strength $Y$ vs. strain rate $\dot{\varepsilon}$ for $\mathrm{TiB}_{2}$ and $\mathrm{B}_{4} \mathrm{C}[16]$. Coupled with similar work with $\mathrm{Al}_{2} \mathrm{O}_{3}$ and $\mathrm{Si}_{3} \mathrm{~N}_{4}$ [17], there is now a complete set of these data for all materials listed in Table 1. However, except for $S i C$, the change in $Y$ from $\dot{\varepsilon}=10^{-4}$ to $10^{3} \mathrm{~s}^{-1}$ does not exceed $0.8 \mathrm{GPa}$. This small change, coupled with the large uncertainties in the data at $10^{3} \mathrm{~s}^{-1}$, make it difficult to use these curves to determine the rate-dependence parameter $D$ [1]. At present, the best way to determine $D$ is to fit the shape of the initial shock-loading portion of at least one wave profile. These values are listed as $D_{\text {profile }}$ in Table 1 . They are different from the values for $D$ in [1] due to an unfortunate error in units conversion in the original paper.

The consequences of this error are three-fold. First, the model no longer reproduces the $Y$ vs. $\dot{\varepsilon}$ data for $\mathrm{SiC}$, the only material with enough change in $Y$ to be a sensitive test. This implies that the assumption in [1] that $Y$ in compression is similar to the fracture stress in tension is not valid. On the other hand, a positive consequence is that the value of $\dot{\varepsilon}$, at which the maximum contribution to $Y$ due to rate dependence occurs, is now of order $10^{5} \mathrm{~s}^{-1}$. This is a reasonable strain rate at the HEL. Using the older, larger values for $D$, this strain rate was too small, being of order $10^{3} \mathrm{~s}^{-1}$. An additional positive consequence is that $D$ is now much closer to the value calculated from Grady's model (i.e., $D_{\mathrm{est}}=\left(3 \rho_{0} C_{0} K_{\mathrm{IC}}\right)^{1 / 3}$, than it was in [1].

One future endeavor is to recalculate all the wave profiles using the exact Grady model, i.e., using $D_{\text {est }}$ in place of $D$, and also setting the other rate-dependence parameter $n$ (see [1]) equal to $1 / 3$ rather than 0.366 . If this produces successful simulations of the data, then two more parameters in the model $(D$ and $n)$ can be determined a priori.

\section{ACKNOWLEDGMENTS}

I would like to thank Dr. Dennis Grady of the Sandia National Laboratory, Albuquerque, with whom I had many valuable discussions, and who also graciously gave me access to much of his unpublished data. Thanks are also due to Dr. David Benson of UCSD who pointed out the conversion error in $D$ in [1].

\section{REFERENCES}

[1] Steinberg D., J. de Physique IV, Coll. C3, suppl., (1991) C3-837-844.

[2] Grady D., Unpublished Data, Sandia National Laboratories 1993.

[3] Steinberg D., Equation of State and Strength Properties of Selected Materials, Lawrence Livermore National Laboratory Report UCRL-MA-106439 (1991).

[4] Rosenberg Z., Brar N. and Bless S., J. Appl. Phys. 70 (1991) 167-171.

[5] Kondo K., Sawaoka A., Sato K. and Ando M., "Shock compression and phase transformation of AIN and BP, "Shock Compression of Condensed Matter, Menlo Park 1981 (AIP, 1982) pp. 325-329.

[6] Kondo K., "Phase transformation of AlN by shock compression", Shock Compression of Condensed Matter-1991, Williamsburg 17-19 June 1991 (Elsevier, 1992) pp. 191-194.

[7] Nakamura A., Mashimo T. and Kodama M., "Shock compression of AlN ceramics," Proc. $6^{\text {th }}$ Int'l Conf. on Mech. Behavior of Mat'ls, Kyoto, 29 July-2 August 1991 (Pergamon, 1991) pp. 395-400.

[8] Grady D., Private Communication, 1992.

[9] Akella J., Unpublished Data, Lawrence Livermore National Laboratory 1991.

[10] Mashimo T., Nakamura A,. Wakamori K. and Miyake M., J. Soc. Mat. Sci., Japan 39 (1990) 1-4.

[11] Grady D. and Kipp M., Shock Compression Properties of Silicon Carbide, Sandia National Laboratories Report SAND92-1832 (1993).

[12] Grady D. and Mashimo T., J. Appl. Phys. 71 (1992) 4868-4874.

[13] Cochran S. and Banner D., J. Appl. Phys. 48 (1997) 2729-2737.

[14] Guinan M. and Steinberg D., J. Phys, and Chem. of Solids 35 (1974) 1501-1512.

[15] Simmons G. and Wang H., Single Crystal Elastic Constants and Calculated Aggregate Properties, 2nd ed. (MIT, 1971) pp. 328-332.

[16] Lankford J, Unpublished Data, Southwest Research Institute 1992.

[17] Lankford, J., J. Mat. Sci. 20 (1985) 53-59.

[18] Munson D. and Lawrence R., J. Appl. Phys. 50 (1979) 6272-6282.

[19] Marsh S., compiler, LASL Shock Hugoniot Data (Univ. of Calif., 1979) p. 260.

[20] Ahrens T., Gust W. and Royce E., J. Appl. Phys. 39 (1968) pp. 4610-4616.

[21] Engineering Property Data on Selected Ceramics, Nitrides. Metals and Ceramics Information Center Report MCIC-HB-07-Vol. 1 (Battelle, 1987) p. 5.3.3-10.

[22] Lankford J., J. Mat. Sci. 18 (1983) 1666-1674. 